\title{
The role of pit-1 in the regulation of the rat growth hormone-releasing hormone receptor gene transcription by glucocorticoids
}

\author{
Haruo Nogami, Yoshiki Hiraoka1, Kiyomoto Ogasawara, Sadakazu Aiso' \\ and Setsuji Hisano \\ Department of Neuroendocrinology, Institute of Basic Medical Sciences, University of Tsukuba, 1-1-1 Tennoudai, Tsukuba, Ibaraki 305-8575, Japan \\ ${ }^{1}$ Department of Anatomy, School of Medicine, Keio University, Tokyo 160-8582, Japan
}

(Requests for offprints should be addressed to H Nogami; Email: hnogami@md.tsukuba.ac.jp)

\begin{abstract}
Glucocorticoids are involved in the regulation of the rat growth hormone-releasing hormone (GHRH) receptor gene expression, but they act only in the presence of the pituitary specific transcription factor, pit-1. In this study, the role of pit-1 in the glucocorticoid stimulation of the GHRH-receptor gene transcription was examined. The results suggest the presence of a silencer element in the promoter and it is postulated that pit-1 permits glucocorticoid action through suppressing the inhibitory effect of an as yet unknown factor that binds to this element. The present results also suggest that the synergistic activation of the rat GHRH-receptor gene transcription depends on the proper distance between the proximal glucocorticoid response element and the pit-1 binding site.
\end{abstract}

Journal of Molecular Endocrinology (2005) 35, 477-488

\section{Introduction}

Glucocorticoid receptor (GR), one of the members of the nuclear receptor super-family, is present in most mammalian tissues and mediates widespread regulatory actions of corticosteroids basically in a cell-specific manner (Truss \& Beato 1993). Ligand-activated GRs bind to the specific DNA element, the glucocorticoid response element (GRE), to induce a transcription of the target gene (Becker et al. 1986). Glucocorticoid responsive genes, in general, contain single or multiple GREs associated with binding sites for other classes of transcription factors to form a hormone response unit. The co-operation of GR with other transcription factors (Schule et al. 1988, Suh \& Rechler 1997) is considered to be important not only for glucocorticoid action but also to restrict action of the steroid within a particular cell type when GR co-operates with tissue-specific transcription factors.

The expression of the growth hormone-releasing hormone $(\mathrm{GHRH})$-receptor is regulated by a number of hormonal or non-hormonal signals such as thyroid hormones (Miki et al. 1995, Korytko \& Cuttler 1997), glucocorticoids (Seifert et al. 1985a,b, Lam et al. 1996, Miller \& Mayo 1997a, Nogami et al. 1999), GHRH (Horikawa et al. 1996, Aleppo et al. 1997, Miller \& Mayo 1997 b), retinoic acids (Nogami et al. 2000) and estrogens (Lam et al. 1996). Within these factors, glucocorticoids are of particular importance, because they trigger the onset of GHRH-receptor expression in the developing pituitary gland (Nogami et al. 1999), which establishes functional correlation between brain and pituitary $\mathrm{GH}$ cells. In a previous study, we identified two GREs, a thyroid hormone response element (TRE) and a binding site for pit-1, a pituitary specific transcription factor (Bodner et al. 1988, Ingraham et al. 1988), in the upstream region of the rat GHRH-receptor gene (Nogami et al. 2002). Pit-1 plays a principal role in the cell type specification of prolactin (PRL), growth hormone $(\mathrm{GH})$ and a subset of thyroid stimulating hormone (TSH) cells, and is a prerequisite for the transcriptional activation of the genes that are specifically expressed in these cells (Bodner et al. 1988, Ingraham et al. 1988), including GHRH-receptor gene (Lin et al. 1992, Iguchi et al. 1999, Miller et al. 1999, Nogami et al. 2002). However, pit-1 alone may not be able to induce expression of pituitary-specific genes. Several lines of evidence suggest this - for example, GHFT1 cells, a clonal cell line of pituitary progenitor that express pit-1 do not express any pituitaryspecific genes other than pit-1 (Lew et al. 1993). The role of pit-1 in the cell type-specific expression of a gene is, therefore, likely to be to permit the induction of the gene expression by an extracellular signal such as hormones or cytokines. The details of this mechanism are mostly unknown.

In this study, we examined the functions of each GRE and the pit-1 element in the transcriptional activation of 
the rat GHRH-receptor gene and the results suggest the presence of a negative regulatory element in the promoter region of this gene. It is conceivable that there is some protein interacting with this element that inhibits GR-dependent gene transcription in the absence of pit-1, and pit-1 probably excludes the inhibitory effects of this protein to permit glucocorticoid to activate gene transcription.

\section{Materials and methods}

\section{Plasmid preparation}

The expression plasmid for the rat GR, pit-1, and thyroid hormone receptor (TR) $\beta 2$ have been described previously (Nogami et al. 2002). The expression plasmid for retinoic acid receptor (RXR) $\alpha$ was a gift from Dr Goda (Department of Nutrition, School of Food and Nutritional Sciences, University of Shizuoka, Shizuoka, Japan). The expression plasmid for CCAAT-enhancer binding protein (C/EBP) $\beta$ (NFIL6 (Akira et al. 1990)) was obtained from Riken BRC DNA Bank, Ibaraki, Japan. The reporter construct prGHRH-R-125/+95luc was a pGL2 (Promega, Madison, WI, USA)-based plasmid carrying a 220 base pair promoter fragment (from +95 to -125 , relative to the transcription start site; Nogami et al. 2002). The promoter of the herpes simplex virus thymidine kinase (from +29 to -107 ; tk) was PCR amplified and cloned into the BglII and HindIII sites of pGL3-basic plasmid (Promega) to generate the pGL3-tk plasmid. The promoter fragment from -115 to -40 of the rat GHRH-receptor gene (Fig. 1A), a minimal fragment that encompasses two GREs (distal GRE (dGRE) and proximal GRE (pGRE)), a TRE and a pit- 1 site, was PCR amplified using primer A (5'-AACGCGTGCTGAGGACACAG-3') and primer B (5'-GGGCATCTGAATATTGAAC- $\left.3^{\prime}\right)$ by a standard procedure. The PGR product was cloned in a pCR2 1 plasmid (Invitrogen, Carlsbad, CA, USA) and the fragment cut out by $\mathrm{KpnI}$ and XhoI was ligated into the pGL3-tk to make a dGpG-p construct. Similarly, the fragments containing pGRE and pit-1 or dGRE and pGRE were prepared by PGR and cloned into pGL3-tk to make pG-p or dGpG plasmid respectively. The fragment with the dGRE and pit-1 site was generated by annealing an oligonucleotide containing dGRE sequences (5'-CTGCTGAGGACACAGAGTCGC-3', dGRE sequence is underlined) with an oligonucleotide carrying the pit-1 sequence (5'-GCTGAATATTGA ACAGAAATGGGACTCTGT-3', pit-1 consensus is underlined), and filling in by Taq polymerase (Takara Shuzo, Shiga, Japan). The resultant fragment was transferred to pGL3-tk to make the dG-p plasmid. Pairs of sense and antisense strands of oligonucleotides spanning dGRE, pGRE or pit-1 elements were annealed and the resultant double stranded DNA fragments were placed upstream of the tk promoter to generate $\mathrm{dG}, \mathrm{pG}$ and $\mathrm{p}$ plasmids respectively. Partial deletion or insertion of the sequences between the pGRE and pit- 1 sites in the dGpG-p plasmid was achieved by generating a mutated PCR fragment using primer A and downstream primers with mutations (see Fig. 5). The PCR fragments with 3- or 7-base pair deletions or a 7-base pair insertion were cloned in pGL3-tk to make dGpG $\Delta 3 \mathrm{p}, \mathrm{dGpG} \Delta 7 \mathrm{p}$ and $\mathrm{dGpG}+7 \mathrm{p}$ respectively. Primers with mutations 5'-G GTGAATA TTGAACGTAGGTCAGGGACATT-3' (mutated sequences are underlined and the pit- 1 site is boxed) or 5'-G GTGACTC TTGAACAGGATGGTGG GACATT-3' (mutated sequences are underlined and the pit-1 site is boxed) was used to introduce mutations into 3 -flanking sequences of the pGRE or pit-1 site respectively. The PGR fragment obtained by primer A and one of these was placed upstream of the tk promoter to generate dGpGmp or dGpG-pm respectively. Similarly, the primers carrying pit- 1 sequences of the rat GH gene (rGH-2, Andersen \& Rosenfeld 1994, 5'-CTGATGG ATAATTTAAAGGATGGTGGGACATT-3'), the rat prolactin gene (rPrl-1P, Andersen \& Rosenfeld 1994, 5'-CATGAATATATATATAAATGGTGGGACATT-3') and the human GHRH-receptor gene (hGHRHR-P2, Iguchi et al. 1999, 5'-CGCTGAATATTCAGGAGGAT GGTGGGACATT-3') were used to generate dGpGGHp, dGpG-PRLp and dGpG-hGHRHRp respectively (see Fig. 6). The replacement of binding sequences of $\mathrm{C} / \mathrm{EBP}$ for the pit- 1 element was achieved by using an antisense primer, 5'-GGATTGGGCAATCGAGGATG GTGGGACATT-3' (C/EBP consensus is underlined). Conversion of two GREs in the dGpG-p plasmid to a consensus GRE (cGRE, GGTACAnnnTGTTCT) was achieved by annealing the following two oligonucleotides; sense, 5'-GGTACACAGTGTTCTATTTGGGG CTGGCAGGTACACAATG, and antisense, 5' - GTGA ATA TTGAACAGGATGGTAGAACATTGTGTAGG TGCC (GRE consensus is underlined and the pit-1 consensus is boxed), filling in and cloned in pGL3-tk (cGcG-p). Similarly, pairs of oligonucleotides 5'-GGT ACAAAATGTTCTGG and 5'-G CTGAATA TTGAA CAGGATGCCAGAACATTTT, or GGTACACAGT GTTGTATTTGGGGGTGGC and AGAACATTGT GTACCTGCCA (consensus for pit-1 was boxed and for GRE was underlined) were used to generate cG-p or cGcG plasmids respectively. All constructs were sequenced by the method of Sanger et al. (1977) prior to the transfection experiments.

\section{Cell culture and transient transfection experiments}

The rat GH cell line, MtT/S (Inoue et al. 1990) and Cos-7 cells, a kidney fibroblast cell line from African green monkey, were maintained in DMEM/F12 medium containing $10 \%$ horse serum, $2 \cdot 5 \%$ fetal bovine 

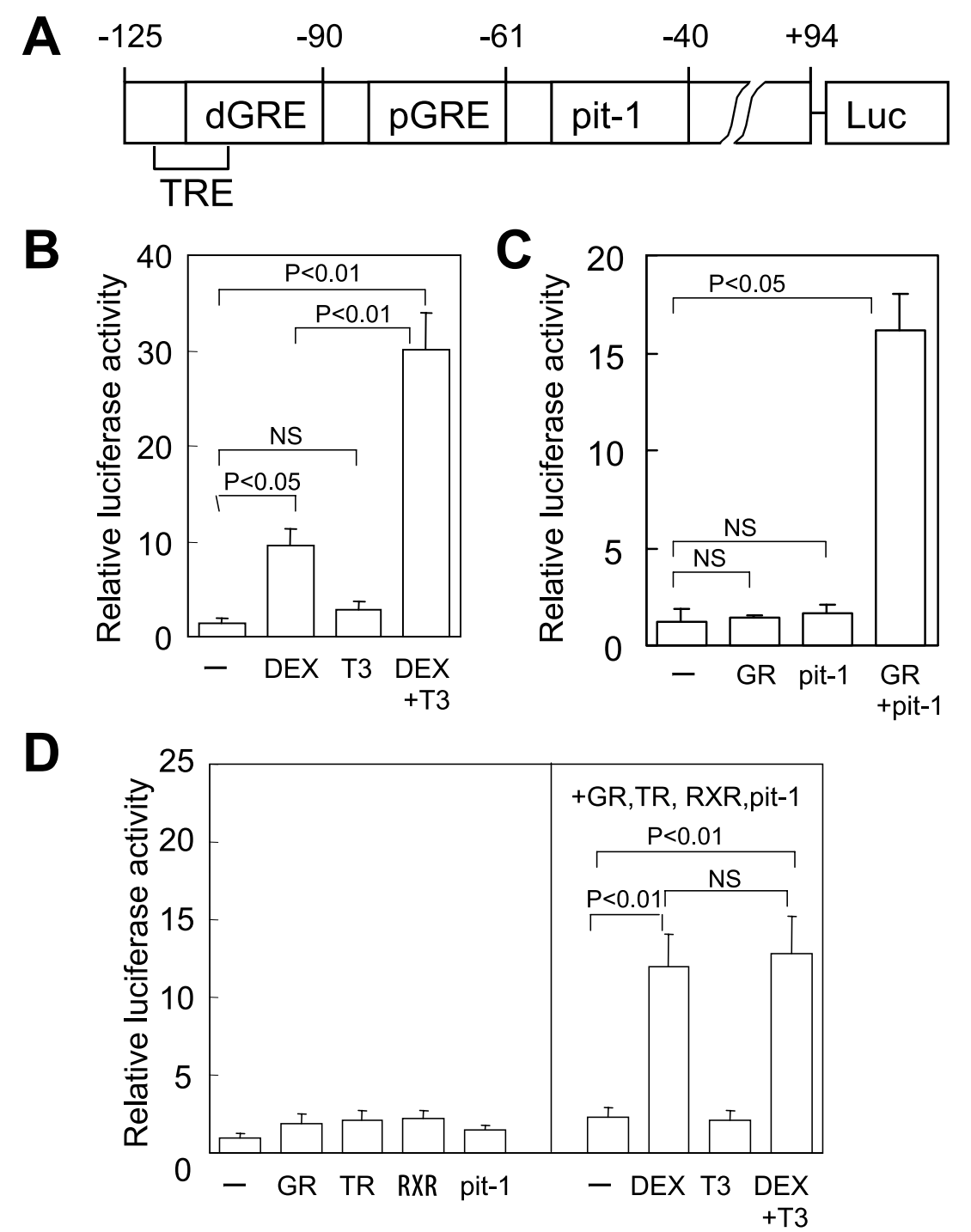

Figure 1 Synergistic activation of the rat GHRH-receptor gene transcription by GR and pit-1. (A) Structure of the prGHRH-R-125/+95-Luc plasmid. The numbers on top indicate the distance from the transcription initiation site. dGRE, distal GRE; pGRE, proximal GRE; pit-1, pit-1 binding site; TRE, thyroid hormone response element (partially overlapping with dGRE), Luc, luciferase. (B) MtT/S cells were transfected with prGHRH-R-125/+95-Luc (500 ng). After transfection, the medium was replaced with fresh medium without hormone $(-)$ or that containing DEX $(100 \mathrm{nM})$ and/or T3 $(1 \mathrm{nM})$, and the luciferase activity of the cell lysate was determined after 24-h incubation. DEX clearly enhanced the reporter gene transcription. T3 augmented the effect of DEX, while it showed little effect alone. (C) Cos-7 cells were transfected with the prGHRH-R-125/+95-Luc (500 ng) alone (-) or in conjunction with the expression plasmid for pit-1 (30 ng) and/or GR (10 ng). In this and the following figures, the total amount of the plasmid DNA used for the transfection was made equal by the addition of pcDNA3 plasmid. After transfection, the cells were incubated with $100 \mathrm{nM}$ DEX for $24 \mathrm{~h}$, and the luciferase activity of the cell lysate was determined $24 \mathrm{~h}$ later. Pit-1 synergized with GR in the induction of the reporter gene transcription. (D) Left panel: Cos-7 cells were transfected with the prGHRH-R-125/+95-Luc (500 ng) and one of pit-1 (30 ng), GR (10 ng), TR 32 (TR, $100 \mathrm{ng}), \mathrm{RXR} \alpha(\mathrm{RXR}, 100 \mathrm{ng})$ or pcDNA3 (-) for the control. After transfection, the cells were incubated with fresh hormone-free medium. Right panel: Cos-7 cells were transfected with the prGHRH-R-125/+95-Luc $(500 \mathrm{ng})$ in conjunction with pit-1, GR, TR 32 and RXR $\alpha$, and then incubated with the medium containing DEX (100 nM) and/or T3 $(1 \mathrm{nM})$. Co-transfection of pit-1, GR, TR $\beta 2$, or $\mathrm{RXR} \alpha$ alone did not significantly increase the basal activity of prGHRH-R-125/+95-Luc construct (left panel). In the Cos-7 cells receiving a combined transfection of GR, pit-1, TRß2 and RXR $\alpha$ (right panel), reporter gene transcription was increased in response to DEX as observed in MtT/S cells, but the synergy between DEX and T3 was not observed. The values are means \pm S.E.M. from 3 (B, $C$ and left panel of $D$ ) or 4 (right panel of D) independent experiments each carried out in triplicate. The data were analyzed by ANOVA followed by Fisher's PLSD test. NS, not significant. 
serum (Gibco-BRL, Rockville, MD, USA) and antibiotics (control medium). For the transfection studies, cells $\left(5 \times 10^{4}\right.$ cells/well $)$ were grown in a 48-well plate with $0.3 \mathrm{ml}$ control medium for $24 \mathrm{~h}$ and on the day of the experiment, the medium was replaced with serum-free Opti-MEM medium (Gibco-BRL). The plasmids were introduced into the cells by a lipofection reagent (Lipofectamine 2000, Gibco-BRL). The amount of the plasmid used per well was $500 \mathrm{ng}$ for reporter constructs, $10 \mathrm{ng}$ for GR, $30 \mathrm{ng}$ for pit-1, $100 \mathrm{ng}$ for TR $\beta 2$ and RXR $\alpha$. The doses of the expression plasmids were determined through preliminary experiments to be the smallest doses that induce a maximal effect, but do not induce non-specific activation of the reporter gene in pGL3 or pGL3-tk. The phRL-GMV (10 ng, Promega) was used to monitor the efficacy of transfection. The transfection was carried out in a serum-free Opti-MEM overnight, and then the medium was replaced with fresh serum-free Opti-MEM with or without hormones (dexamethasone (DEX) $100 \mathrm{nM}$ and/or triiodothyronine (T3) $1 \mathrm{nM}$ ), followed by an additional 24-h incubation. After incubation, luciferase activity of the cell lysate was determined using a dual-luciferase reporter assay system (Promega). The luciferase activity derived from the reporter construct was normalized for that of Renilla luciferase of phRL-CMV co-transfected for internal standard, and expressed as percent of Renilla luciferase activity.

\section{Statistical analyses}

The data are expressed as means \pm S.E.M. of 3-5 independent experiments each carried out in triplicate, and the significance of difference was determined by Student's $t$-test or a one-way analysis of variance (ANOVA) followed by Fisher's protected least significant difference (PLSD) test.

\section{Results}

The reporter gene expression from the prGHRH-R125/+95-Luc construct which has two GREs, a TRE and a binding site for pit-1 (Fig. 1A) was increased by DEX treatment in $\mathrm{MtT} / \mathrm{S}$ cells, a $\mathrm{GH}$ cell line expressing GHRH-receptor (Fig. 1B). While thyroid hormone (T3) alone had little effect on the reporter gene transcription from this construct, it clearly enhanced the effect of DEX. The reporter gene transcription from prGHRH-R-125/+95-Luc in the presence of DEX did not show a significant increase with the expression of pit-1 or GR alone in Cos-7 cells (Cos-7 does not express pit-1 and GR); however, it showed a 13-fold increase over the control when these two were co-transfected (Fig. 1G). In Cos-7 cells to which GR (10 ng), TR $\beta 2$ (100 ng), RXR $\alpha \quad(100 \mathrm{ng})$ and pit-1 (30 ng) were co-transfected, DEX induced the reporter gene expression from prGHRH-R-125/+95-Luc as well as in $\mathrm{MtT} / \mathrm{S}$ cells (Fig. 1D, right panel); however, a synergy between T3 and DEX was not observed. In the control experiment, T3 up-regulated the rat GH promoter $(+55$ to -240 , relative to the transcription initiation site)-directed reporter gene transcription in Cos-7 cells co-transfected with TR $\beta 2, \operatorname{RXR} \alpha$, and pit- 1 (2.7-fold increase by T3, $P<0.05$ vs without T3 by Student's $t$-test, $n=3)$. The basal activity of GHRH-R-125/+95Luc in Cos-7 cells did not show any significant increase with the expression of GR, TR $\beta 2, \operatorname{RXR} \alpha$ or pit-1 (Fig. 1D, left panel). These results indicate that the synergistic activation of the rat GHRH-receptor gene transcription by glucocorticoid and pit- 1 observed in the GH cell line, MtT/S cells, is reproducible in Cos-7 cells. The results also suggest that some of the factors required for thyroid hormone action on the rat GHRH-receptor promoter are missing in Cos-7 cells.

In order to elucidate the role of GR and pit-1 in rat GHRH-receptor gene transcription, we examined the enhancer activity of two GREs and the pit- 1 binding site of this gene, using heterologous thymidine kinase promoter and Cos-7 cells. First, the effects of transfection of the expression plasmid for pit-1 and GR on the basal activity of pGL3 basic or pGL3-tk plasmid were examined (Fig. 2A and B). Up to $100 \mathrm{ng}$ GR or pit- 1 expression plasmid did not affect reporter gene expression from pGL3 (Fig. 2A). Transcription of reporter gene from pGL3-tk was also not affected by GR but was significantly enhanced by $100 \mathrm{ng}$ pit- 1 expression plasmid (Fig. 2B).

As shown in Fig. 2C, neither dGRE nor pGRE showed distinct GRE activity alone. However, the reporter activity increased by $4 \cdot 4$ times in response to DEX when these two GREs were combined (dGpG in Fig. 2C). When non-consensus GREs in $\mathrm{dGpG}$ were converted to consensus GRE (cGcG in Fig. 2C), reporter gene expression increased by 4.6 times in response to DEX. Thus, a pair of GHRH-receptor GREs appears to compose a functional glucocorticoid response unit, its activity being almost compatible with that of the $\mathrm{cGcG}$ construct.

When dGpG-p with two GREs and a pit-1 site (Fig. 3A) was transfected to Cos-7 cells in conjunction with expression plasmids for pit- 1 and GR, the reporter gene expression from this construct was enhanced by DEX by about 6-6-fold (Fig. 3B, bottom panel). However, interestingly, this construct showed minimal response to DEX without pit-1 (1.6-fold, Fig. 3B, top panel). This latter finding apparently conflicts with the results of Fig. 2 that the transcription from dGpG with no pit- 1 site responded to DEX by $4 \cdot 4$-fold (Fig. 2), while it coincides well with previous results that showed that the transcription of the GHRH-receptor gene requires pit-1 (Lin et al. 1992, Iguchi et al. 1999, Miller et al. 1999, Nogami et al. 2002). The only explanation possible for this discrepancy is that some DNA 

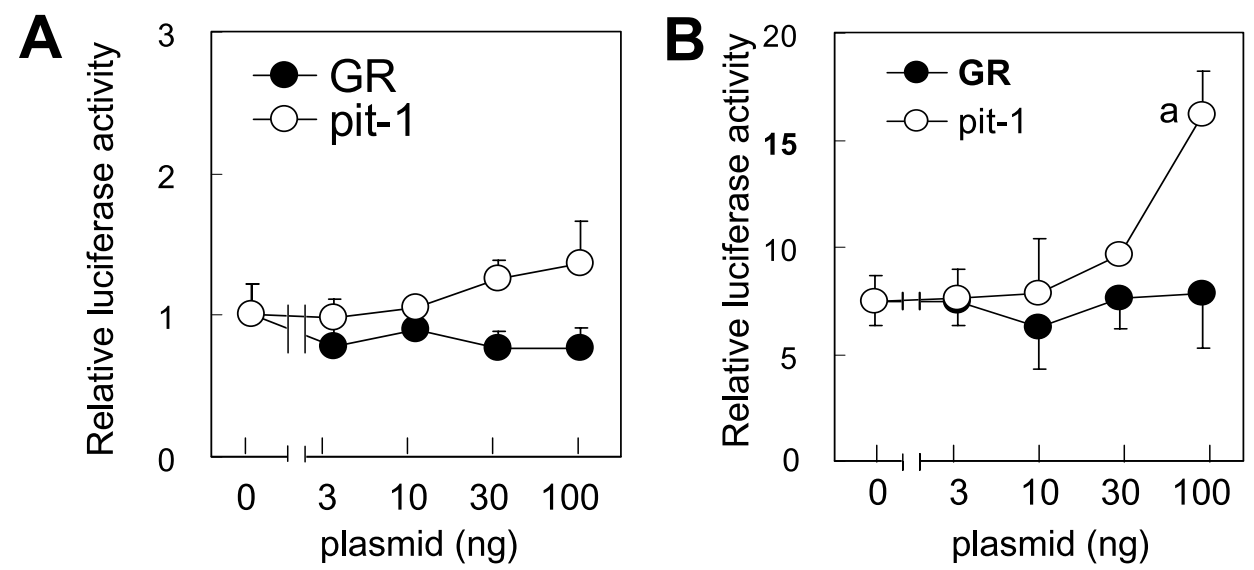

Relative luciferase activity

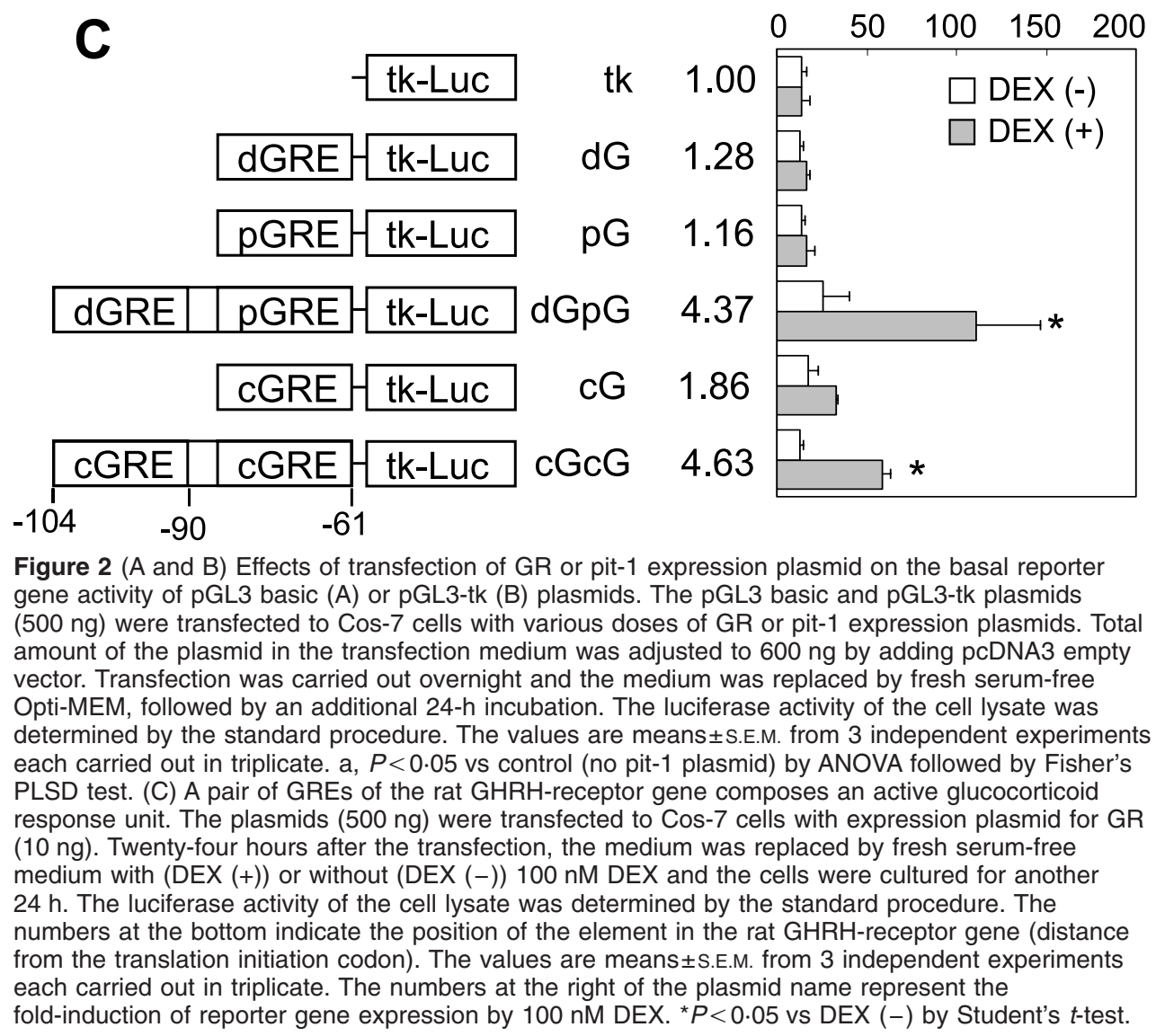

sequences within dGpG-p that are absent in $\mathrm{dGpG}$, the pit-1 site and the 14-base pair spacer between pGRE and the pit-1 site, contain the silencer element. Since GRE-pit-1 constructs (dG-p or pG-p in Fig. 3) did not react to DEX, this suggests that two GREs are necessary for the synergy of glucocorticoids and pit-1. A single consensus GRE-pit-1 construct (cG-p) also weakly responded to DEX in the presence of pit-1. On the other hand, the construct with two cGREs (cGcG-p) showed about a $12 \cdot 8$-fold increase in reporter expression in response to DEX in the presence of pit-1. Unlike the dGpG-p, transcription from cGcG-p increased in 


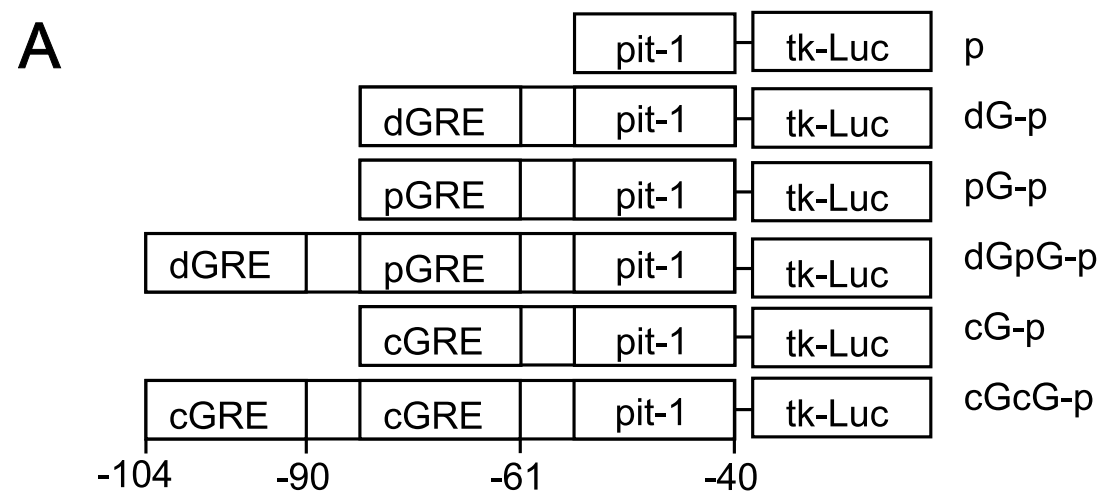

B

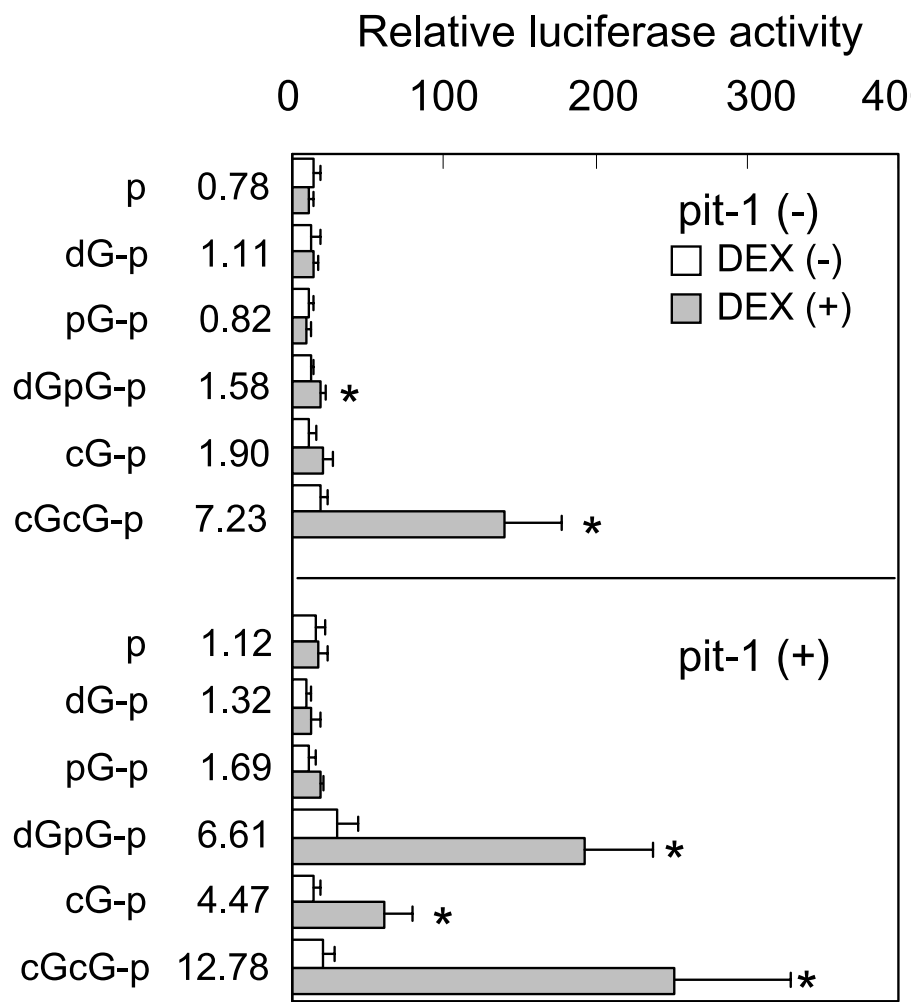

Figure 3 Effects of pit-1 on the reporter gene expression from constructs carrying promoter with pit-1. In this and the following figures, the plasmids illustrated in (A) $(500 \mathrm{ng})$ were transfected to Cos-7 cells with expression plasmid for GR (10 $\mathrm{ng}$ ) alone (B, top panel) or GR and pit-1 (30 ng) (B, bottom panel). Total amount of the plasmid in transfection was adjusted to $540 \mathrm{ng}$ using empty vector, pcDNA3. After $24 \mathrm{~h}$ transfection, the medium was replaced by fresh serum-free medium with (DEX (+)) or without (DEX (-)) $100 \mathrm{nM}$ DEX and the cells were cultured for another $24 \mathrm{~h}$. The luciferase activity of the cell lysate was determined by the standard procedure. The numbers in $(A)$ indicate the position of the element in the rat GHRH-receptor gene (distance from the translation initiation codon). Values are means \pm S.E.M. from 3 independent experiments each carried out in triplicate. The numbers at the right of the plasmid name in (B) represent the fold-induction of reporter gene expression by $100 \mathrm{nM}$ DEX. ${ }^{*} P<0.05$ vs DEX $(-)$ by Student's $t$-test.

response to DEX by about $7 \cdot 2$-fold without pit- 1 (Fig. 3B), although this construct contains a putative silencer element (Fig. 3A).
The hypothesis that the pit-1 site or the 14-base pair sequence between pit-1 and pGRE harbors a silencer element was evaluated in the experiments shown in 


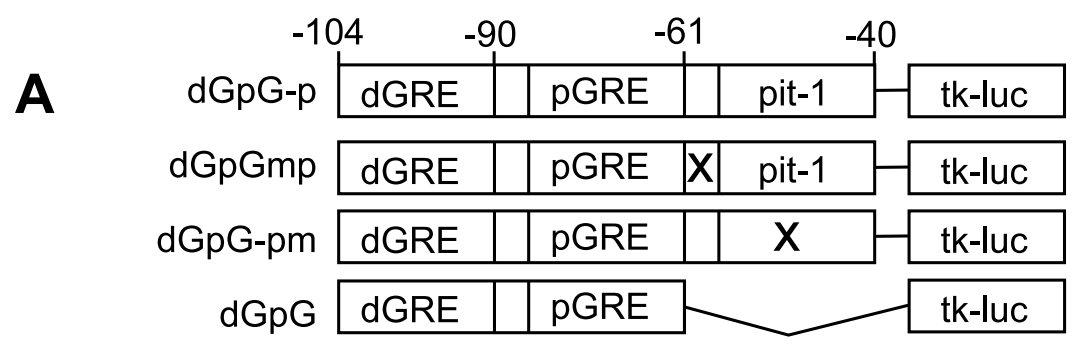

B

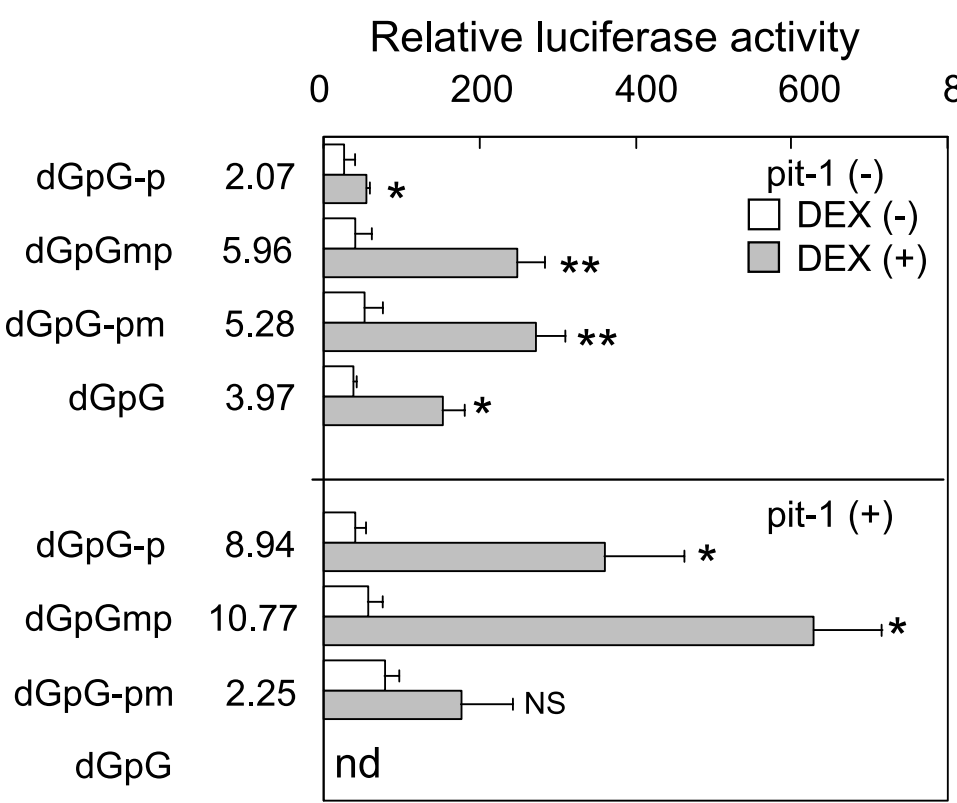

Figure 4 Effects of mutations of a putative silencer element on the reporter gene expression from the dGpG-p construct. Transfection and determination of the promoter activity were carried out as described in Fig. 3. Values are means \pm S.E.M. from 3 (pit-1 (+)) or 4 (pit-1 (-)) independent experiments each carried out in triplicate. The numbers at the right of the plasmid name in (B) represent the fold-induction of reporter gene expression by $100 \mathrm{nM}$ DEX. nd, not determined. ${ }^{\star} P<0.05 ;{ }^{*} P<0.01$ vs DEX (-) by Student's $t$-test. NS, not significant vs DEX $(-)$. nd, not done.

Figs 4 and 5. When a mutation was introduced to 7-base pair sequences 3 '-flanking to the pGRE (dGpGmp in Fig. 4), the pit-1 independent DEX induction of the promoter was observed. The expression of pit-1 further enhanced the activity of this construct. Unexpectedly, a mutation of the pit-1 element, which has previously been shown to eliminate pit-1 activity (dGpG-pm in Fig. 4) also resulted in pit-1-independent DEX induction. However, the further enhancement of the DEX-induced reporter gene activity by pit-1 was not observed in this construct, in agreement with our previous results showing that this mutation diminishes the effect of DEX in the GH-producing MtT/S cells (Nogami et al. 2002). These data suggest that the putative silencer element spans both the 14-base pair spacer and the pit-1 element. This result coincides with the fact that the deletion of these sequences $(\mathrm{dGpG})$ results in a distinct pit-1-independent DEX induction. In the experiment illustrated in Fig. 5, we examined the effect of deletion of part of the silencer element. The deletion was made at the 5 '-end, so that the pit- 1 binding site remained intact. Unlike the mutation of this region (Fig. 4), the deletion of 3 or 7 bases of the 14-base pair spacer between the pit- 1 site and pGRE (dGpG $\Delta 3 p$ and dGpG $\Delta 7 \mathrm{p}$ in Fig. 5) did not result in a marked change in the reporter gene activity in the absence of pit-1. Unexpectedly, the effect of pit-1 became less evident with the 3-base pair deletion and was not observed any more with the 7-base pair deletion. This result is in contrast to that shown in Fig. 4, showing that the synergy was still observed in 


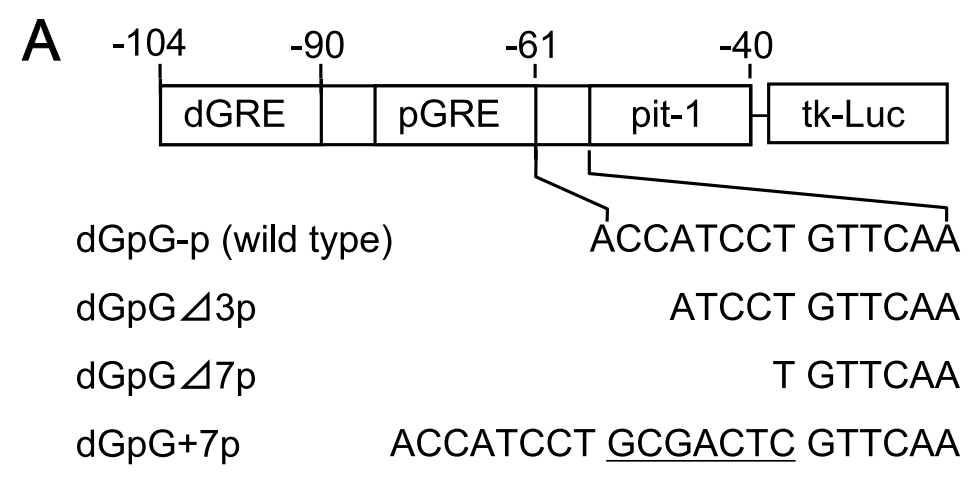

B

Relative luciferase activity

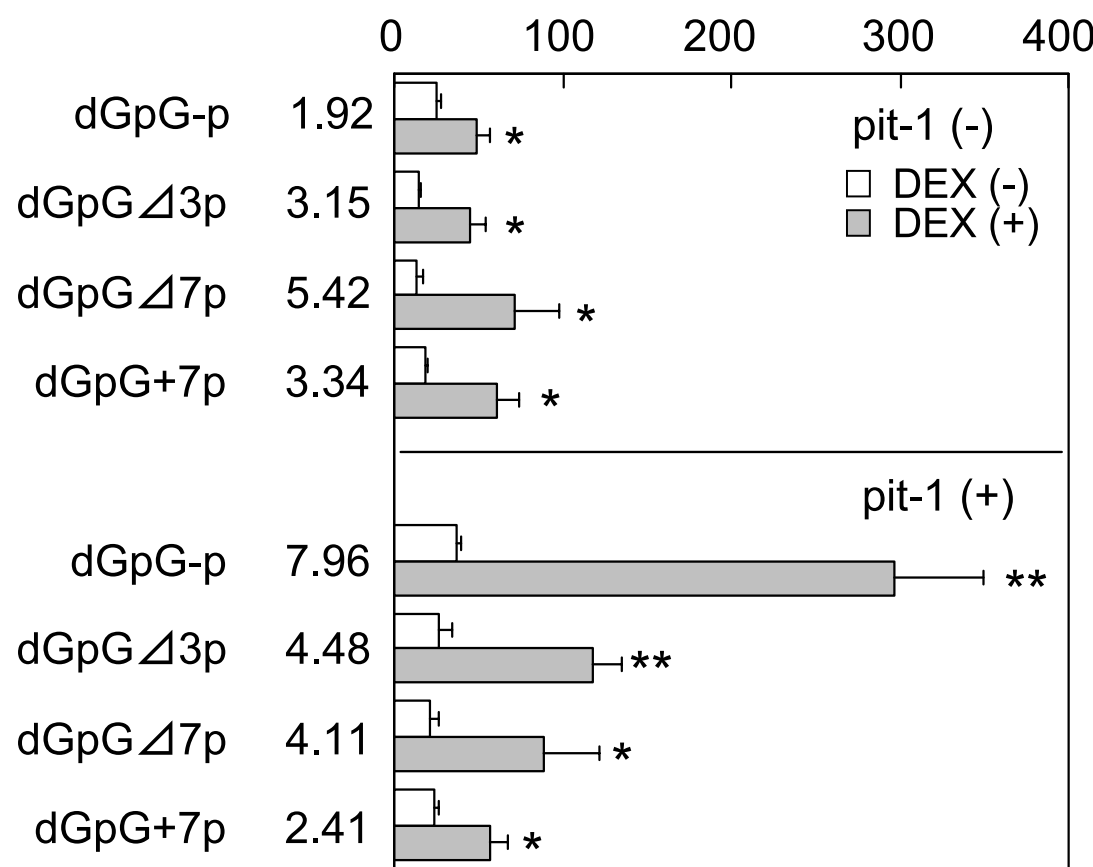

Figure 5 Effects of partial deletion of a putative silencer element or a 7-base-pair insertion (underlined) to it on the reporter gene expression from the dGpG-p construct. Transfection and determination of the promoter activity were carried out as described in Fig. 3. Values are means \pm S.E.M. from 5 independent experiments each carried out in triplicate. The numbers at the right of the plasmid name in $(B)$ represent the fold-induction of reporter gene expression by $100 \mathrm{nM} D E X .{ }^{*} P<0.05 ;{ }^{\star \star} P<0.01$ vs DEX (-) by Student's t-test.

dGpGmp construct where the 7-base pair element was mutated without changing the distance from pit-1 to pGRE (Fig. 4). The synergy was also not seen in the construct in which the pit-1 element was placed 21 base pairs downstream of pGRE $(\mathrm{dGpG}+7 \mathrm{p})$, the distance being normally 14 base pairs (Fig. 5), while this construct responded to DEX without pit- 1 .

Next we examined whether the pit-1 element of the GHRH-receptor gene as the binding site for pit-1 and the as yet unknown silencer protein can be replaced by different pit-1 elements of other pit-1-dependent genes. Three pit-1 elements, rat GH2, rat Prl-1P and human GHRHR-P2, have consensus pit-1 sequences, ATGnATA(T/A)(T/A) (Andersen \& Rosenfeld 2001) in the lower sequences as the rat GHRH-receptor gene. These elements were placed at the position of the pit-1 element of dGpG-p so that the distance between pGRE and the pit- 1 consensus was not changed. As shown in 
A

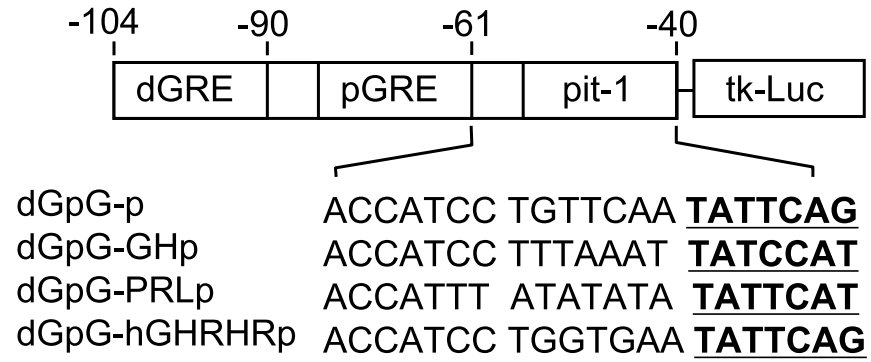

B
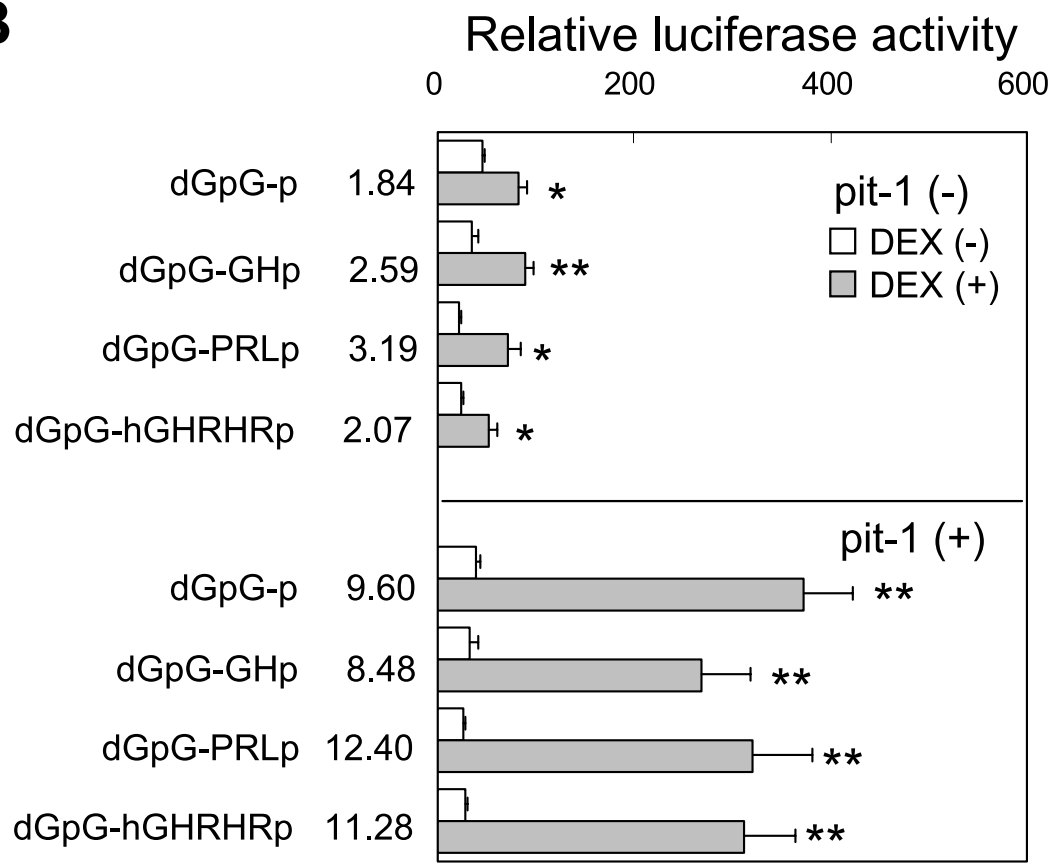

Figure 6 Pit-1 elements of the other pit-1-dependent genes function similarly to that of the rat GHRH-receptor gene. The pit-1 binding site of dGpG-p was replaced with that of rat GH (rat GH-2), rat prolactin (rat prl-1p) or human GHRH-receptor (hGHRHR-P2) promoter to generate dGpG-GHp, dGpG-PRLp and dGpG-hGHRHRp respectively. Transfection and determination of the promoter activity were carried out as described in Fig. 3. Values are means \pm S.E.M. from 4 independent experiments each carried out in triplicate. The numbers at the right of the plasmid name in $(B)$ represent the fold-induction of reporter gene expression by 100 nM DEX. ${ }^{\star} P<0.05 ;{ }^{*} P<0.01$ vs DEX (-) by Student's $t$-test.

Fig. 6, these three pit-1 elements functioned similarly to that of the rat GHRH-receptor gene.

In order to ascertain whether or not other classes of transcription factor can replace pit-1, the pit-1 element of dGpG-p was replaced by the consensus $\mathrm{C} / \mathrm{EBP}$ binding site to make dGpG-cebp (Fig. 7). This construct responded to DEX treatment without co-transfection of $\mathrm{C} / \mathrm{EBP} \beta$ or pit- 1 expression plasmid, probably because the pit-1 binding site was eliminated. Pit-1 expression did not affect the glucocorticoid responsiveness of this construct but additional expression of $\mathrm{C} / \mathrm{EBP} \beta$ (NFIL6) enhanced the glucocorticoid effect by about $2 \cdot 5$-fold. The expression of $\mathrm{G} / \mathrm{EBP} \beta$ did not induce glucocorticoid-dependent transcription of reporter gene in dGpG-p.

\section{Discussion}

The results from the present study suggest that two GREs and a pit-1 element in conjunction with a putative silencer element, newly postulated in this study, compose a pit-1-dependent glucocorticoid response unit in the rat GHRH-receptor gene. Although the mechanism is still obscure, the binding of an unknown substance to this region is considered to suppress the glucocorticoid action 
$\mathbf{A}$

\begin{tabular}{|c|c|c|c|c|}
\hline & $04 \quad-c$ & & & 0 \\
\hline GpG-cebp & dGRE & pGRE & C/EBP & tk-Luc \\
\hline$d G p G-p$ & dGRE & pGRE & pit-1 & tk-Luc \\
\hline
\end{tabular}

B

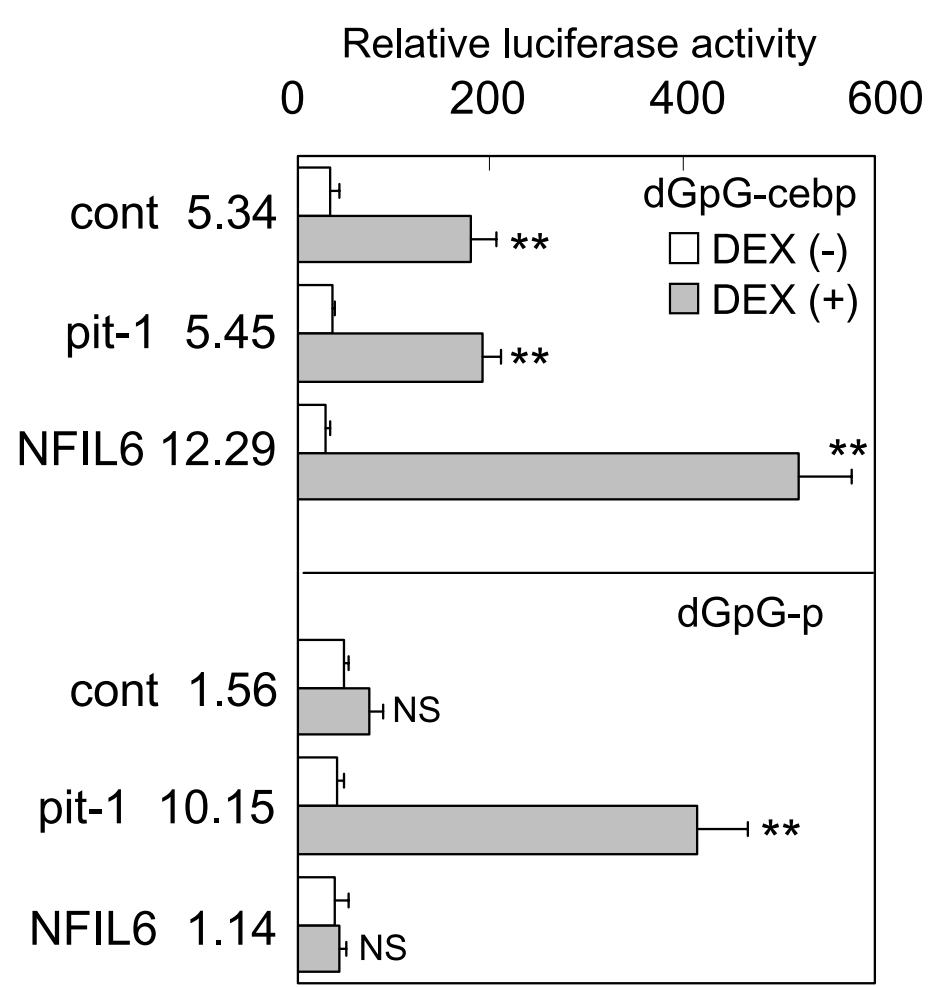

Figure 7 Effects of the replacement of the pit-1 element by a C/EBP consensus sequence on the promoter activity. The reporter constructs were transfected with expression plasmid for GR (10 ng) and $30 \mathrm{ng}$ pcDNA3, expression plasmid for pit-1 or NFIL6 (C/EBP $\beta)$, and the effect of DEX on the reporter gene activity was determined as described in Fig. 3. Values are means \pm S.E.M. from 3 independent experiments each carried out in triplicate. The numbers at the right of the plasmid name in $(\mathrm{B})$ represent the fold-induction of reporter gene expression by $100 \mathrm{nM}$ DEX. ${ }^{\star \star} P<0.01$ vs DEX $(-)$ by Student's $t$-test. NS, not significant; cont, control.

through dGRE and pGRE. The binding of pit-1 to its element possibly released these GREs from suppression, resulting in glucocorticoid-dependent gene transcription. This is a novel function of pit-1, which restricts the glucocorticoid-dependent induction of the GHRHreceptor gene transcription that is mediated by the hormone receptors expressed ubiquitously to pit-1expressing cells. Since thyroid hormone action on this gene depends upon glucocorticoids (Nogami et al. 2002), it appears that pit- 1 is also implicated in the cell-specific action of thyroid hormone on the GHRH-receptor gene transcription.
The results shown in Figs 2 and 3 suggest that 21-base pair sequences $3^{\prime}$ to the pGRE (this region contains the pit-1 site) are responsible for silencing transcription driven by the combined two GREs in the rat GHRH-receptor gene. The mutation of the pit-1 element (dGpG-pm in Fig. 4) resulted in pit-1independent reporter gene expression by DEX to a level similar to that of the $\mathrm{dGpG}$ plasmid in which the entire silencer element was eliminated. These results suggest that the pit-1 site is the principal part of the silencer element; however, the 7-base pair sequence next to the pGRE also appears to be involved in the silencer 
element because mutation (dGpGmp in Fig. 4) or deletion (dGpG $\Delta 7$ in Fig. 5) of this sequence resulted in the pit-1-independent DEX induction of reporter. Thus a putative silencer element encompasses the 21-base pair sequences from the the 3 -end of the pGRE to the pit-1 consensus.

It is possible that the binding of a certain protein to this silencer element blocks the binding of GR to neighboring pGRE. Since binding of GRs to both GREs is required for the glucocorticoid action (Fig. 2), probably because of co-operative binding of receptors to adjacent GREs as demonstrated previously (Schmid et al. 1989, Tsai et al. 1989), inhibition of GR binding to pGRE alone by a silencer protein should result in a significant negative effect on gene transcription. It is also likely that the silencer protein does not interfere with the binding of GR to GREs, but inhibits the formation of the complex of DNA-bound GRs and various other classes of nuclear proteins. The DNA-bound GRs have been shown to bind nuclear coactivators such as GRIP1 (Hong et al. 1996), and further recruitment of modulators that have histone acetylase or methylase activity is proposed to be required for glucocorticoiddependent gene transcription. In the presence of pit-1, such as in pit-1-expressing cells, preferential binding of pit-1 to its element probably removes the silencer protein to allow GRs to interact with pGRE, or the formation of the complex of GRs and other nuclear proteins, leading to the activation of the transcription.

The synergistic activation of the reporter gene expression by DEX and pit-1 was seen in dGpGmp (Fig. 4), but not in $\mathrm{dGpG} \Delta 7 \mathrm{p}$ or in $\mathrm{dGpG}+7 \mathrm{p}$ (Fig. 5). These results indicate that the distance between pGRE and the pit-1 site is critical, suggesting that a physical interaction between GR and pit-1 may be involved in the mechanism responsible for this synergy. Our results also suggest that the synergy is not an intrinsic function of GREs and the pit-1 element of the rat GHRHreceptor promoter, because the replacement of consensus GRE for both dGRE and pGRE or other pit-1 elements for that of dGpG-p did not abolish the synergy (Fig. 6).

Little is known about the mechanisms of the synergy of nuclear hormone receptors and pit-1, despite the fact that the synergy may explain why a hormone could regulate a particular gene expression in a cell-specific manner. In the rat prolactin gene, the transcription of which is highly dependent on estrogen, a binding site for estrogen receptor is located near one of the binding sites for pit-1 (1D-site) in the distal enhancer region (Kim et al. 1988). Although this estrogen response element (ERE) is weak and non-consensus, the binding of pit-1 to the 1D-site enables estrogens to regulate PRL gene transcription through this ERE in the pituitary PRL cells (Nowakowski \& Maurer 1994). The role of pit-1 in the rat prolactin promoter appears different from that in the rat GHRH-receptor gene, because the 1D pit-1 site of the rat PRL gene promoter cannot be replaced by other classes of transcription factor binding sites (Nowakowski \& Maurer 1994), while that of the GHRH-receptor promoter can be replaced by the binding site for C/EBP (Fig. 7). The physical association of the receptors for T3 and retinoic acid and pit- 1 is reported to be involved in the synergistic activation of the rat GH transcription (Palomino et al. 1998). However, in the activation of the PRL gene, the interaction of pit-1 and estrogen receptors has not been demonstrated (Nowakowski \& Maurer 1994). As discussed above, the physical interaction of GRs and pit-1 is likely to be involved in the rat GHRH-receptor gene transcription. However, our mammalian two-hybrid assay failed in demonstrating interaction of GR and pit-1, suggesting either that the physical interaction of these two may not be involved in the rat GHRH-receptor transcription, or that the interaction is too weak to be detected with our system (data not shown).

In conclusion, the present results suggest that the roles of pit-1 in rat GHRH receptor gene transcription is to eliminate the inhibitory effects of a silencer on GREs, and to synergize with GRs, the latter being dependent on the distance between pGRE and the pit-1 binding site. The nature of the protein $(\mathrm{s})$ that binds to the presumptive silencer region is currently unknown. Since Cos-7 cells, kidney fibroblasts, express this protein, it is likely that the protein is expressed ubiquitously. Identification of the silencer protein is required for the further elucidation of the molecular mechanisms of the cell-specific activation of GHRH-receptor gene transcription by glucocorticoids.

\section{Acknowledgements}

This work was supported in part by the Twenty-First Century COE Program, and the Grant-in-Aid for Scientific Research (C) no. 13670003 and no.15590513 from the Ministry of Education, Science, Sports and Culture, Japan. The authors declare that there is no conflict of interest that would prejudice the impartiality of this scientific work.

\section{References}

Akira S, Isshiki H, Sugita T, Tanabe O, Kinoshita S, Nishio Y, Nakajima T, Hirano T \& Kishimoto T 1990 A nuclear factor for IL-6 expression (NF-IL6) is a member of a C/EBP family. EMBO foumal 9 1897-1906.

Aleppo G, Moskal SF, De Grandis PA, Kineman RD \& Frohman LA 1997 Homologous down-regulation of growth hormone-releasing hormone receptor messenger ribonucleic acid levels. Endocrinology 138 1058-1065.

Andersen B \& Rosenfeld MG 1994 Pit-1 determines cell type during development of the anterior pituitary gland. A model for 
transcriptional regulation of cell phenotypes in mammalian organogenesis. Fournal of Biological Chemistry 269 29335-29338.

Andersen B \& Rosenfeld MG 2001 POU domain factors in the neuroendocrine system: lessons from developmental biology provide insights into human disease. Endocrine Reviewes 22 $2-35$.

Becker PB, Gloss B, Schmid W, Strahle U \& Schutz G 1986 In vivo protein-DNA interactions in a glucocorticoid response element require the presence of the hormone. Nature $\mathbf{3 2 4}$ 686-688.

Bodner M, Castrillo JL, Theill LE, Deerinck T, Ellisman M \& Karin M 1988 The pituitary-specific transcription factor GHF-1 is a homeobox-containing protein. Cell $\mathbf{5 5} 505-518$.

Hong H, Kohli K, Trivedi A, Johnson DL \& Stallcup MR 1996 GRIP1, a novel mouse protein that serves as a transcriptional coactivator in yeast for the hormone binding domains of steroid receptors. PNAS 93 4948-4952.

Horikawa R, Hellmann P, Gella SG, Torsello A, Day RN, Muller EE \& Thorner MO 1996 Growth hormone-releasing factor (GRF) regulates expression of its own receptor. Endocrinology 137 2642-2645.

Iguchi G, Okimura Y, Takahashi T, Mizuno I, Fumoto M, Takahashi Y, Kaji H, Abe H \& Chihara K 1999 Cloning and characterization of the 5 -flanking region of the human growth hormone-releasing hormone receptor gene. Fournal of Biological Chemistry 274 12108-12114.

Ingraham HA, Chen RP, Mangalam HJ, Elsholtz HP, Flynn SE, Lin CR, Simmons DM, Swanson L \& Rosenfeld MG 1988 A tissue-specific transcription factor containing a homeodomain specifies a pituitary phenotype. Cell $\mathbf{5 5}$ 519-529.

Inoue K, Hattori MA, Sakai T, Inukai S, Fujimoto N \& Ito A 1990 Establishment of a series of pituitary clonal cell lines differing in morphology, hormone secretion, and response to estrogen. Endocrinology 126 2313-2320.

Kim KE, Day RN \& Maurer RA 1988 Functional analysis of the interaction of a tissue-specific factor with an upstream enhancer element of the rat prolactin gene. Molecular Endocrinology $\mathbf{2}$ 1374-1381.

Korytko AI \& Cuttler L 1997 Thyroid hormone and glucocorticoid regulation of pituitary growth hormone-releasing hormone receptor gene expression. Fournal of Endocrinology 152 R13-R17.

Lam KSL, Lee MF, Tam SP \& Srivastava G 1996 Gene expression of the receptor for growth-hormone-releasing hormone is physiologically regulated by glucocorticoids and estrogen. Neuroendocrinology 63 475-480.

Lew D, Brady H, Klausing K, Yaginuma K, Theill LE, Stauber C, Karin M \& Mellon PL 1993 GHF-1-promoter-targeted immortalization of a somatotropic progenitor cell results in dwarfism in transgenic mice. Genes and Development 7 683-693.

Lin C, Lin SC, Chang CP \& Rosenfeld MG 1992 Pit-1 dependent expression of the receptor for growth hormone releasing factor mediates pituitary cell growth. Nature $360765-768$.

Miki N, Ono M, Murata Y, Ohsaki E, Tamitsu K, Ri T, Demura H \& Yamada M 1995 Thyroid hormone regulation of gene expression of the pituitary growth hormone-releasing factor receptor. Biochemical and Biophysical Research Communications 217 1087-1093.

Miller T \& Mayo K $1997 a$ Glucocorticoids regulate pituitary growth hormone-releasing hormone receptor messenger ribonucleic acid expression. Endocrinology 138 2458-2465.
Miller TL \& Mayo KE $1997 b$ Growth hormone-releasing hormone (GHRH) receptor mRNA regulation in rat pituitary cells. Program of the 79th Annual Meeting of the Endocrine Society, Minneapolis, MN, P1-79 (Abstract).

Miller TL, Godfrey PA, Dealmeida VI \& Mayo KE 1999 The rat growth hormone-releasing hormone receptor gene: structure, regulation, and generation of receptor isoforms with different signaling properties. Endocrinology 140 4152-4165.

Nogami H, Inoue K, Moriya H, Ishida A, Kobayashi S, Hisano S, Katayama M \& Kawamura K 1999 Regulation of growth hormone-releasing hormone receptor mRNA expression by glucocorticoids in MtT-S cells and in the pituitary gland of fetal rats. Endocrinology $1402763-2780$.

Nogami H, Matsubara M, Harigaya T, Katayama M \& Kawamura K 2000 Retinoic acids and thyroid hormone act synergistically with dexamethasone to increase growth hormone-releasing hormone receptor mRNA expression. Endocrinology 141 4396-4401.

Nogami H, Hiraoka Y, Matsubara M, Nonobe E, Harigaya T, Katayama M, Hemmi N, Kobayashi S, Mogi K, Aiso S, Kawamura K \& Hisano S 2002 A composite hormone response element regulates transcription of the rat GHRH receptor gene. Endocrinology 143 1318-1326.

Nowakowski BE \& Maurer RA 1994 Multiple Pit-1-binding sites facilitate estrogen responsiveness of the prolactin gene. Molecular Endocrinology 8 1742-1749.

Palomino T, Sanchez-Pacheco A, Pena P \& Aranda A 1998 A direct protein-protein interaction is involved in the cooperation between thyroid hormone and retinoic acid receptors and the transcription factor GHF-1. FASEB Fournal 12 1201-1209.

Sanger F, Nicklen S \& Coulson AR 1977 DNA sequencing with chain-terminating inhibitors. PNAS 74 5463-5467.

Schmid W, Strahle U, Schutz G, Schmitt J \& Stunnenberg H 1989 Glucocorticoid receptor binds cooperatively to adjacent recognition sites. EMBO foumal 8 2257-2263.

Schule R, Muller M, Kaltschmidt C \& Renkawitz R 1988 Many transcription factors interact synergistically with steroid receptors. Science 242 1418-1420.

Seifert H, Perrin M, Rivier J \& Vale W 1985a Binding sites for growth hormone releasing factor on rat anterior pituitary cells. Nature 313 487-489.

Seifert H, Perrin M, Rivier J \& Vale W $1985 b$ Growth hormone-releasing factor binding sites in rat anterior pituitary membrane homogenates: modulation by glucocorticoids. Endocrinology $117424-426$.

Suh DS \& Rechler MM 1997 Hepatocyte nuclear factor 1 and the glucocorticoid receptor synergistically activate transcription of the rat insulin-like growth factor binding protein-1 gene. Molecular Endocrinology 11 1822-1831.

Truss M \& Beato M 1993 Steroid hormone receptors: interaction with deoxyribonucleic acid and transcription factors. Endocrine Revierws 14 459-479.

Tsai SY, Tsai MJ \& O’Malley BW 1989 Cooperative binding of steroid hormone receptors contributes to transcriptional synergism at target enhancer elements. Cell $\mathbf{5 7} 443-448$.

Received 5 September 2005

Accepted 20 September 2005

Made available online as an Accepted Preprint 4 October 2005 